\title{
Investigación
}

\section{El personaje de Hugo Chávez en Isla Presidencial. Un análisis de la sátira política a través de la teoría de las representaciones sociales de Serge Moscovici ${ }^{1}$}

\section{The character of Hugo Chávez in Presidential Island. An analysis of political satire through the theory of social representations of Serge Moscovici}

Daniela Alejandra Santofimio Prada ${ }^{*}$

Diana Sofía Sosa Bautista**

Recepción: 27/04/2020 • Aprobación: 02/09/2020 • Publicación: 01/12/2020

Para citar este artículo

Santofimio Prada, D. A., \& Sosa Bautista, D. S. (2020). El personaje de

Hugo Chávez en Isla Presidencial. Un análisis de la sátira política a través de la teoría de las representaciones sociales de Serge Moscovici. Dos mil tres mil, 22, e232. https://doi.org/10.35707/dostresmil/22232

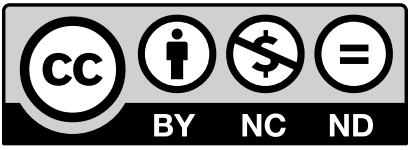

\footnotetext{
${ }^{1}$ El presente artículo se deriva del trabajo de grado Sedientos de poder, los personajes de la Isla Presidencial. Un análisis de la sátira política, Programa Ciencia Política, Universidad de Ibagué.

*Programa de Ciencia Política, Universidad de Ibagué, Colombia. Correo electrónico: danielaasp822@gmail.com

"* Programa de Ciencia Política. Universidad de Ibagué, Colombia. Correo electrónico: dsofiasosa98@gmail.com
} 
Resumen. En este artículo se realiza un análisis de contenido a la serie audiovisual Isla Presidencial desde la Ciencia Política, a través de las representaciones sociales planteadas por Serge Moscovici, basado en el personaje de Hugo Chávez como jefe de Estado latinoamericano. El objetivo de este escrito es poder establecer por medio de las representaciones sociales del poder, si el personaje del exmandatario venezolano cumple con la premisa de la sed de poder; de ser así, establecer la manera en la que alcanza y satisface su sed de poder. El análisis del humor y la sátira política se realizará sobre los últimos años de gobierno, aspectos que se relatan y abarcan en los 35 capítulos que conforman la serie Isla Presidencial.

Abstract. In this article a content analysis of the audiovisual series Presidential Island from the Political Science's perspective, through social representations proposed by Serge Moscovici, based on the character of Hugo Chávez as Latin American head of state is performed. The aim of this article is to be able to establish through social representations of power, if the character of the former Venezuelan president meets the premise of craving power, if so, establish the way in which he achieves and satisfies his greed for power. The analysis of political humor and satire will be made on the last years of government, aspects that are related and covered in the 35 episodes that make up Presidential Island, the series.

Palabras claves

Teoría de las representaciones sociales, sátira política, humor político, poder, audiovisual.

Key words

Theory of social representations, political satire, political humor, power, audiovisual. 


\section{Introducción}

Para Moscovici (2000), las representaciones sociales del poder constituyen la base inconsciente de las posiciones socialmente asumidas por las personas tendientes a la conservación del poder, buscan visibilizar la imagen, estereotipo o conocimiento que tiene la sociedad con respecto a este. Así mismo, es importante definir el poder como la capacidad de hacer real lo posible y generar un sentimiento de satisfacción a la persona que lo ejerce (Marina, 2008). Es por esto que el presente documento permitirá entender cómo el personaje de Hugo Chávez, como jefe de Estado al igual que los demás personajes de la serie, ostentan el poder como una herramienta inherente a su cargo, lo cual genera el despliegue de acciones populistas desde su estilo, ideología, discurso y estrategias, para la preservación del mismo.

Para analizar con detalle las representaciones sociales del poder a través de la sátira y el humor político visualizado en Isla Presidencial (2010), se tomará como referente a Serge Moscovici (1961), quien establece tres dimensiones de la representación social, dentro de las cuales aparece la información, el campo de representación y la actitud. La primera de ellas, se refiere al conocimiento previo que tienen los sujetos sobre una particularidad específica, que como consecuencia se refleja en caracteres de estereotipos.

El personaje de Hugo Chávez representado en la Isla Presidencial propone estereotipos de su imagen, ideología, personalidad, que no necesariamente son reales, sino que han sido construidos desde la misma comunidad que lo ha elegido para que ostente el poder. La segunda dimensión es el campo de representación, aquí se organiza el contenido de la representación en forma jerarquizada. Denise Jodelet (1989) establece que se debe realizar un estudio a partir del contenido representativo; es decir, todo aquello que constituye las representaciones y las estructuras a través de las cuales se reflejan los sistemas de representación; en razón a esto, su estudio será la base para poder determinar el sentido, lo que significa, los procesos y las formas de organización del contenido. La última dimensión propuesta por Moscovici (1961) es la actitud, la cual es una de las más importantes debido a que refleja la posición que se toma frente a la representación, que puede resultar ser favorable o desfavorable hacia el objeto de la representación social, en este caso cada uno de los personajes de la serie. Hugo Chávez tiene una posición muy visible para la audiencia en cuanto al concepto de poder.

El humor político es un medio por el cual se hace burla a las formas de gobierno de quienes ostentan el poder, mostrando un lenguaje sin censura, en el que prevalece la crítica (Ulloa, 2008). Históricamente en Colombia, el humor político se ha hecho por medio de caricaturas, la radio y el periódico, pero con el advenimiento y la innovación de la tecnología, en la época de los años noventa el humor político empezó por medio de la televisión. Este recurso ha sido importante para demostrar toda clase de inconformidad con las irregularidades que se presentan en los gobiernos, frente a la manera en que se ejerce el poder y la política. Un personaje importante en este tema, fue Jaime Garzón para Colombia, quien utilizó el humor político en los programas Zoociedad TV, $i$ Quac! El noticiero, Lechuza y CM\& (El Heraldo, 2016). En efecto,

El personaje de Hugo Chávez en Isla Presidencial.

Un análisis de la sátira política a través de la teoríade las representaciones sociales de Serge Moscovici 
abordaremos el humor que se presenta en la serie audiovisual Isla Presidencial, la cual confronta situaciones y personajes de la política internacional, para satirizar a la política iberoamericana.

La sátira política consiste en criticar varios contextos de la política de un país, no solo la forma en la que gobiernan, sino situaciones o actuaciones que se presentan en los mandatarios de lo que no se considera correcto (Valero, 2014); se realizará el estudio sobre el personaje Hugo Chávez en la serie audiovisual Isla Presidencial identificando su lenguaje, apariencia física, emocional y su lógica de acción, con el propósito de seguir ostentando el poder dentro de la Isla, observando sus ideologías, la manera en la que se muestra ante la ciudadanía y audiencia.

La sátira y el humor político se han desarrollado a través de la plataforma digital YouTube, en el canal Isla Presidencial. Esta serie se inició el 15 de enero de 2010, es una serie venezolana, creada por Oswaldo Graziani y Juan Andrés Ravell; por medio de la sátira política, narra una historia de los personajes que representan presidentes de países hispanoamericanos que naufragan en una isla después de la cumbre iberoamericana de jefes de Estado y de gobierno. Estos deberán luchar por su supervivencia y se verán inmersos en diferentes situaciones que los llevarán a mostrar su sed de poder.

Para desarrollar el presente escrito, se llevó a cabo una investigación con enfoque cualitativo para el análisis de contenido. Sin embargo, el análisis de contenido cuenta también con un enfoque cuantitativo, el cual permitirá establecer por medio de la cuantificación, marcadores de cómo aparecen las representaciones sociales del poder en el personaje de Hugo Chávez, con el registro del número de veces en que se visibiliza, así como las características atribuidas durante los capítulos de Isla Presidencial.

Pretendemos que el estudio que realizamos sea de manera objetiva, por medio del cual se pueda obtener a través de cada una de las etapas de este proceso de análisis: una población, muestra, unidades de análisis, unidades de contexto, categorías, codificación y cuantificación; inicialmente el contenido será analizado con base a las representaciones sociales identificadas en esta unidad documental, que es Isla Presidencial. Para el presente escrito, llevaremos a cabo el análisis de la totalidad de los capítulos de la serie de YouTube, Isla Presidencial, en la que hace presencia el personaje objeto de estudio, Hugo Chávez.

\section{Hugo Chávez, el Mesías}

¿Es posible afirmar que el exmandatario venezolano Hugo Chávez es el verdadero mesías de la Isla Presidencial? Los capítulos 9 y 11 de la segunda temporada de la serie audiovisual llamados Mesías parte 1, y Mesías parte 2, relatan cómo el expresidente Hugo Chávez hace milagros como parte de una manipulación ejercida por el exmandatario estadounidense, Barack Obama.

Para entender las relaciones de poder del personaje Hugo Chávez desde la sátira política, se estudian las características principales y radicales de los Estados con ideologías de izquierda, toda vez que él pertenece a esta corriente; en medio de su lenguaje, discurso y expresiones, es fácil establecer las aptitudes de sus enemigos capitalistas. Asimismo, alrededor de su personaje se desatan historias y problemas que involucran a la mayoría de presidentes de Isla Presidencial.

El personaje de Hugo Chávez en Isla Presidencial.

Un análisis de la sátira política a través de la teoríade las representaciones sociales de Serge Moscovici 
A través de este escrito abordaremos la sátira hacia el imperialismo, las conspiraciones, los discursos frecuentes que el exmandatario venezolano solía expresar y su figura de líder, esto permitirá demostrar las dimensiones que establece Moscovici (1961): la información, el campo de representación y la actitud. Finalmente, se analizará cómo los productores de la serie acogen al personaje de Hugo Chávez como un robot y con esto logran mostrar otras relaciones de poder diferentes a las que se dan dentro de las dinámicas de la serie Isla Presidencial.

\section{2. Érase una vez volcanes, cocos y monos imperialistas}

Hugo Chávez en vida se caracterizó por las fuertes críticas que dirigía frecuentemente hacia el sistema capitalista, a los países que promueven dicho sistema, a los Estados que lo ejecutan y a todo aquel que hiciera parte de este. Por lo anterior, Graziani y Ravell (2010), durante el desarrollo de la serie Isla Presidencial, no dudaron en expresar ese sentimiento antimperialista. Para esta oportunidad, su conflicto con el capitalismo se ve reflejado en diversas ocasiones, principalmente en discusiones con los expresidentes de Colombia, en un primer momento con el personaje de Álvaro Uribe Vélez y posteriormente Juan Manuel Santos, los cuales serán detallados en el transcurso de este escrito.

Érase una vez volcanes, cocos y monos imperialistas, no solo es una crítica al imperialismo, sino a las frecuentes conspiraciones que el expresidente, ya fallecido, Hugo Chávez, proclamaba en escenarios nacionales e internacionales. Las conspiraciones contienen una de las tantas historias detalladas en Isla Presidencial, como una forma de obtener, mantener y vivir el poder.

Con lo anterior, queremos abarcar los dos conceptos que utiliza Hugo Chávez de imperialismo y conspiración, para demostrar las relaciones de poder no solo de él, sino también de los otros presidentes iberoamericanos que hacen parte de la serie Isla Presidencial y que, en medio de las conversaciones, problemas y soluciones, se caracteriza su sed de poder.

\section{Chávez vs. Imperialismo}

No sería extraño que en Marte haya habido civilización, pero a lo mejor llegó allá el capitalismo, llegó el imperialismo y acabó con ese planeta. (Hugo Chávez, 2011).

Si bien este trabajo va de la mano con la sátira política, la anterior frase no es broma ni hace parte de la serie Isla Presidencial. El expresidente Hugo Chávez nunca intentó ocultar su inconformismo hacia el sistema capitalista. En el año 2011, durante su discurso en el Día Mundial del Agua, manifestó su odio radical hacia el capitalismo, queriendo dar a entender que esta corriente económico-política es capaz de acabar cualquier forma de vida (Expansión.mx, 2011). Lo anterior es un ejemplo de las constantes luchas frente al imperialismo como causa de los problemas sociales mundiales adjudicados por el exmandatario venezolano.

El personaje de Hugo Chávez en Isla Presidencial.

Un análisis de la sátira política a través de la teoríade las representaciones sociales de Serge Moscovici 
El personaje del expresidente Hugo Chávez durante su aparición en la serie audiovisual Isla Presidencial hace constantes críticas al imperialismo, refiriéndose al sistema social, político y económico de diferentes países de América, especialmente Estados Unidos y Colombia. Las críticas establecidas por el personaje de Hugo Chávez se dirigieron a la explotación social y económica que ejercen los países que poseen este régimen. Cabe resaltar que el imperialismo es la fase histórica superior al capitalismo (Lenin, 2003); sin embargo, en Isla Presidencial se consideran como conceptos iguales.

El mandato presidencial de Hugo Chávez que abarcó desde el año 1999 hasta su fallecimiento en el año 2013, se caracterizó por ser propulsor del movimiento socialista, que empezó a formarse a partir de la segunda mitad del siglo xIx. Para Hinkelammert:

Los movimientos socialistas [...] se originaron por la crítica del capitalismo. En ellos el pensamiento teórico juega un papel fundante, a pesar de que su origen real es la experiencia de explotación vivida por las capas populares en el sistema capitalista. (Hinkelammert, 2003, p. 12).

Es aquí el factor detonante sobre el cual yace el constante juzgamiento del capitalismo por parte de Hugo Chávez a la conformación de estructuras sociales dentro del sistema capitalista, el sacrificio dentro del mismo de las clases obreras y masas populares, y las bonanzas para una minoría de la población.

El conflicto de Chávez vs. Imperialismo (que se ha estudiado como capitalismo) no fue el único enfrentamiento que tuvo durante su hegemonía política; a su vez, durante las presidencias vigentes de él como jefe de Estado de Venezuela y Álvaro Uribe Vélez como presidente de Colombia, cada uno en diferentes escenarios políticos, señalaban los errores y hacían fuertes acusaciones sobre su homogéneo.

Para el episodio 4 de la primera temporada, surge un escenario en el cual los dos bandos, alienados (jefes de Estado con ideología de derecha) y no alineados (jefes de Estado con ideología de izquierda), se enfrentan en un partido de fútbol para saber cuál equipo podrá salir de la isla utilizando una balsa construida por ellos mismos. Durante el juego, el equipo de los alineados hace un gol, dejando de mal humor al mandatario venezolano. De esta manera, se desata una discusión dentro de la cual el exjefe de Estado venezolano y el exmandatario colombiano se gritan los defectos del uno al otro:

— Álvaro Uribe Vélez: ¡Socialista!

— Hugo Chávez: ¡Capitalista!

— Álvaro Uribe Vélez: ¡Comunista!

- Hugo Chávez: ¡Fascista!

— Álvaro Uribe Vélez: ¡Oportunista! (Min 2:09, Capítulo 4, Temporada 1)

El personaje de Hugo Chávez en Isla Presidencial.

Un análisis de la sátira política a través de la teoríade las representaciones sociales de Serge Moscovici 
Figura 1. La disputa (Isla Presidencial, Capítulo 4, Temporada 1)

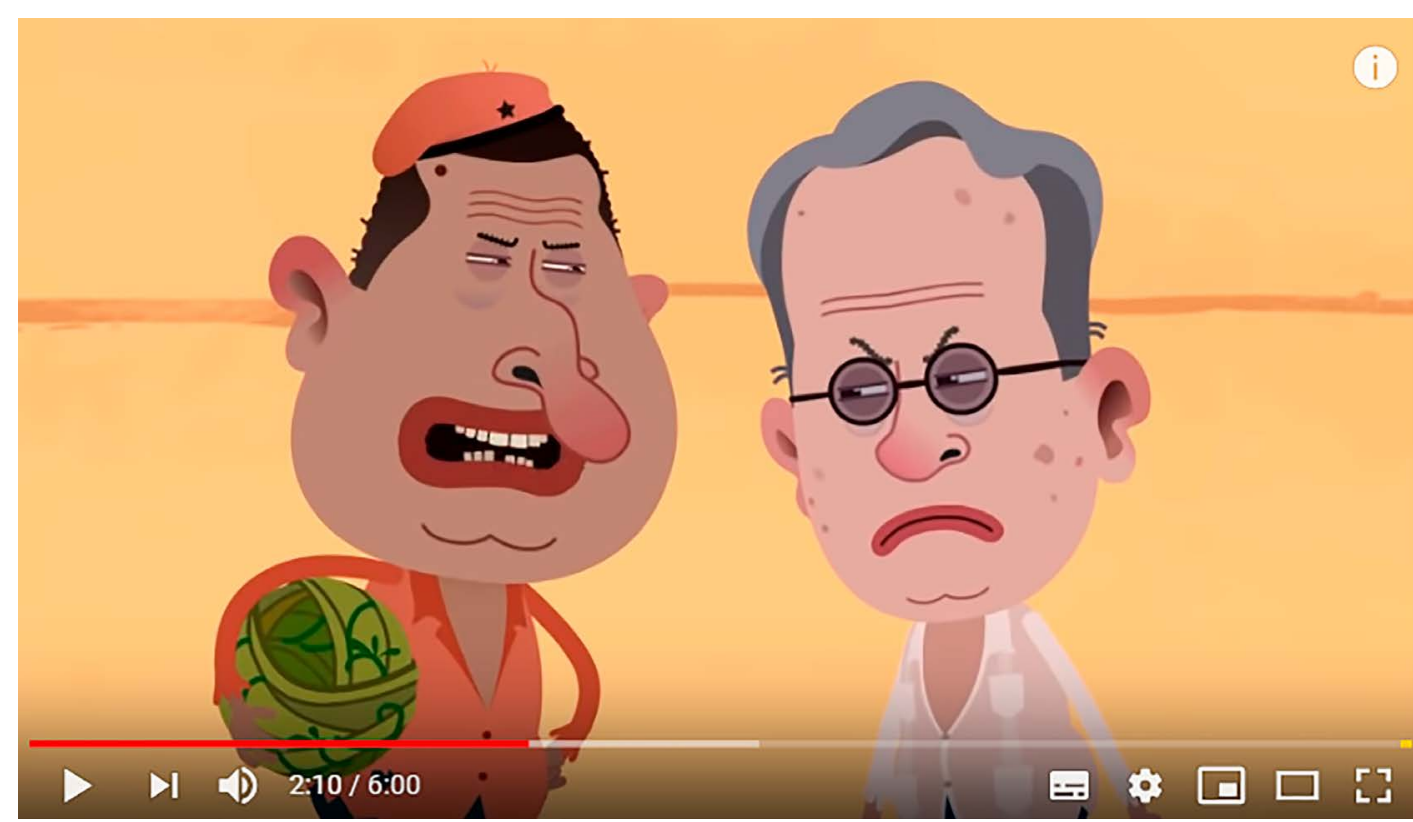

De derecha a izquierda: Álvaro Uribe Vélez (exjefe de Estado de Colombia); Hugo Chávez (exjefe de Estado de Venezuela Fuente: Isla Presidencial [YouTube] (11 de julio, 2010)

En la disputa (Figura 1) se pueden evidenciar características adjudicadas a ambos personajes: imperialista y comunista. Cabe resaltar que cada uno de los gobiernos de los presidentes fueron abanderados de estos sistemas y opositores radicales del otro. Durante el gobierno de Hugo Chávez, comprendido entre los años 1999 y 2013 y el de Álvaro Uribe Vélez, desde el año 2002 hasta el 2010, tomaron como referencia los sistemas de gobierno del país vecino para sus respectivas campañas políticas, alusivo a las situaciones negativas como una estrategia para retener popularidad. De esta manera, Uribe siempre empleó las expresiones "oportunista, comunista, socialista", como un símbolo de amenaza y de significado opuesto a la utopía que las personas quieren llegar. Un ejemplo de esto, se presenta en la declaración que Uribe hace durante la presentación de su libro: No hay causa perdida, en la que criticó a Chávez por moverse hacia el comunismo con un disfraz de demócrata (Gómez, 2012). El mandatario Hugo Chávez siempre calificó, criticó e hizo fuertes acusaciones, al gobierno de Álvaro Uribe Vélez y a todo su grupo de bancada llamándolos "fascistas, imperialistas, capitalistas".

Las disputas del capitalismo y socialismo encabezados por los exjefes de Estado Hugo Chávez y Álvaro Uribe Vélez, son parte del constante conflicto teórico entre el régimen capitalista y socialista. Sin embargo, como lo establece Hinkelammert, ambos sustentos buscan un mismo fin: 
El paradigma del capitalismo parte del mercado, para construir su utopía del mercado total idealizado. El paradigma del socialismo parte de las necesidades del ser humano concreto, para desembocar en la idealización de una sociedad sin mercado con su utopía del comunismo. Ambos prometen una solución homogénea y universal para la totalidad de la humanidad, y ambos vinculan su solución con la vigencia de un progreso técnico ilimitado e irrestricto y con la promesa de un esplendor futuro derivado de su magia. (Hinkelammert, 2003, p. 18).

Para la primera temporada en este mismo capítulo, el exmandatario venezolano se reúne con su equipo de los no alineados para crear una táctica y así empatar el partido por el cual se definía quiénes podrían escapar de la isla, es así que, para finalizar su idea, Hugo Chávez dice: "Chávez desafía al capitalismo y Goooool" (minuto 3:19).

Se puede afirmar que durante el $60 \%$ de los capítulos que conforman la primera temporada, se hizo alusión a la sátira del imperialismo por parte del exmandatario venezolano, ya fallecido. A continuación, mostraremos cómo, a través de las conspiraciones, se puede ejercer el poder a través de personajes que están acostumbrados a tenerlo.

\section{Conspiraciones}

¿La conspiración puede ser utilizada como herramienta para alcanzar el poder? En un primer momento nos deberíamos centrar en la definición que propone la Real Academia Española (s.f.) para definir conspirar, allí se estipula que: "1. intr. Dicho de varias personas: Unirse contra su superior o soberano. / 2. intr. Dicho de varias personas: Unirse contra un particular para hacerle daño" (s. p.). Cabe resaltar que esta última definición de conspiración no se hace de manera voluntaria, toda vez que se utilizan vicios en el consentimiento que serán explicados en el transcurso de este capítulo.

Por medio de las conspiraciones se pueden unir voluntades para alcanzar un objetivo, en el caso específico del presente escrito: el poder. El primer ejemplo que traemos a colación se presenta en el primer capítulo de la segunda temporada, titulado El volcán. Hugo Chávez quiere tomar el poder sobre la isla; para esto, idea y lleva a cabo un plan, en el que recurre al miedo para alcanzar su objetivo. De esta manera, expone un problema: el volcán de la isla amenaza con acabar su lugar y con ello terminar la vida de los personajes. Para esto, el expresidente venezolano utiliza al exmandatario Evo Morales, para alcanzar la máxima autoridad de la isla. Evo Morales empieza a realizar rugidos alusivos del volcán, haciéndoles creer que provienen de este $y$, por lo tanto, hay una amenaza.

El personaje de Hugo Chávez en Isla Presidencial.

Un análisis de la sátira política a través de la teoríade las representaciones sociales de Serge Moscovici 
Figura 2. La ballena (Isla Presidencial, Capítulo 1, Temporada 2)

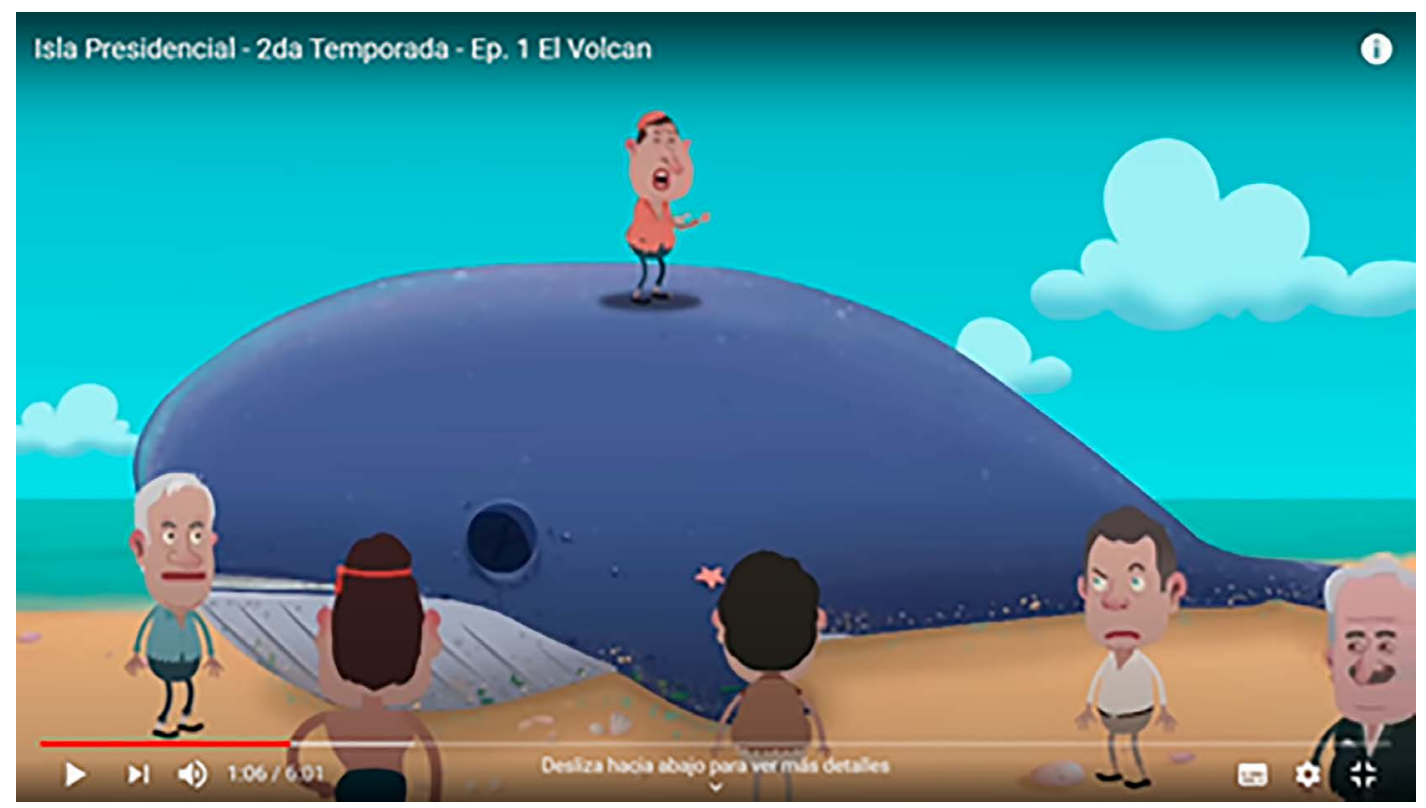

De izquierda a derecha: Daniel Ortega (presidente de Nicaragua), Cristina Fernández (expresidente de Argentina), Sebastián Piñera (presidente de Chile), Rafael Correa (expresidente de Ecuador), Evo Morales (expresidente de Bolivia), Hugo Chávez (expresidente de Venezuela), Juan Carlos I (antiguo Rey de España) y Juan Manuel Santos (expresidente de Colombia).

Fuente: Isla Presidencial 2. ${ }^{a}$ Temporada - Ep. 1 El Volcán [YouTube] (5 de octubre de 2018)

En la anterior imagen, se evidencia el momento en el cual Chávez trata de crear un problema, el cual necesita una solución que él tiene. No obstante, no siempre las conspiraciones dan lugar a nuestro objetivo planteado, y suelen enfrentar, como en la política, opositores que tratan de evitarlas, sabotearlas y detenerlas. Según Edelman, en su libro Construcción del espectáculo político, expresa que:

Es más importante visualizar quién apoya y quién se opone a una determinada propuesta y el cálculo táctico de ese posicionamiento, que entender y precisar de qué se trataba la propuesta en cuestión. Y, sobre todo, qué consecuencias tendrá para el bienestar público. (Edelman, 1991, p. 10).

Durante el periodo de la presidencia de Hugo Chávez, manifestó junto con su par, Evo Morales, sobre las conspiraciones del mundo imperialista. En el año 2011 Chávez aseguró la ausencia de vida en Marte a causa del capitalismo, en el año 2012 manifestó que el 9/11 fue planeado por el expresidente Bush. Por otra parte, el exmandatario boliviano en diferentes ocasiones ha manifestado supuestas conspiraciones y relación entre la calvicie y los alimentos transgénicos, así como la correlación entre comer pollo y ser desviado como hombre. Las anteriores afirmaciones dan lugar a que se puedan atribuir características de imperialista a cualquier situación e inferir que los problemas que existen en el mundo son a causa del capitalismo. 
En el capítulo 1, El volcán, de la segunda temporada, se puede evidenciar cómo el exmandatario colombiano Juan Manuel Santos ejerce resistencia a la conspiración planteada por el expresidente venezolano. En la escena, el jefe de Estado de Colombia exterioriza que en repetidas ocasiones el expresidente venezolano ha inventado conspiraciones sobre diferentes objetos de la isla, y con ello, ha aprovechado para culpar de los problemas al imperialismo:

- Hugo Chávez: como todos ustedes saben, en esta isla hay un volcán imperialista que nos quiere destruir a todos.

- Juan Manuel Santos: Hugo, Hugo, esta no va a ser como tu teoría de conspiración sobre los monos.

- Hugo Chávez: como todos sabemos en esta isla hay unos monos, unos monos imperialistas que nos quieren destruir a todos.

- Juan Manuel Santos: Hugo, Hugo, esta no va a ser como tu teoría de conspiración sobre los cocos.

- Hugo Chávez: como todos sabemos en esta isla hay unos cocos, unos cocos imperialistas que nos quieren destruir a todos. (Min 4:44, Capítulo 1, Temporada 2).

"Érase una vez unos volcanes, cocos y monos imperialistas" es la frase que mejor permite caracterizar a Hugo Chávez en Isla Presidencial. Es una alusión sobre una persona, quien siempre tiene una historia por contar. En su carrera de ser el líder y tener el poder de la isla, el personaje se caracteriza en sus tres temporadas por ser incansable, por siempre hablar, acusar al capitalismo, por la manipulación que ejerce sobre Evo Morales, y por nunca perder la oportunidad de ver conspiraciones del imperialismo en cualquier lugar.

Figura 3. Espantapájaros (Isla Presidencial, Capítulo 4, Temporada 1)

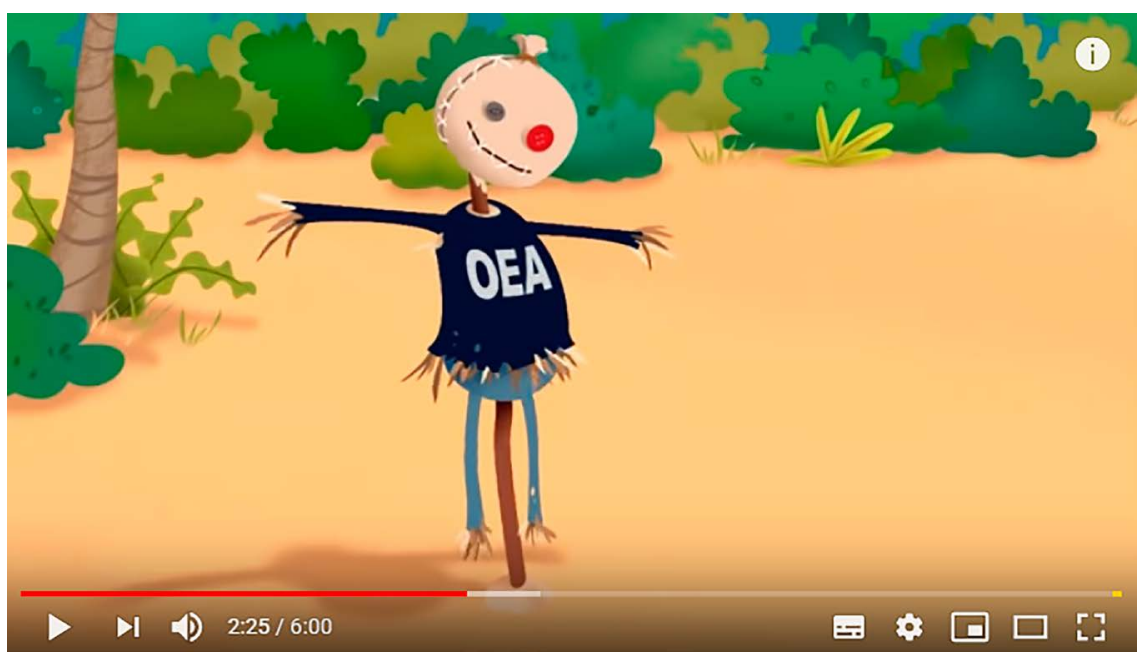

Representación de la Organización de Estados Americanos (OEA)

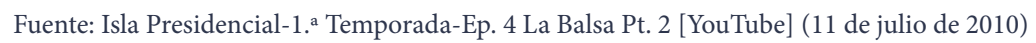

El personaje de Hugo Chávez en Isla Presidencial.

Un análisis de la sátira política a través de la teoríade las representaciones sociales de Serge Moscovici 
Las conspiraciones presentadas en la serie audiovisual no solo se dan entre personajes de la misma, sino que resalta la aparición de instituciones internacionales. La primera es la Organización de Estados Americanos (OEA), en el episodio 4 de la primera temporada, presentan una institución con poco funcionamiento y eficacia dentro de su marco de ejecución. En el capítulo 6 de la primera temporada aparece la Agencia Central de Inteligencia (CIA), en medio de una conversación de los mandatarios Evo Morales y Hugo Chávez en el bosque. Después de una larga caminata por la búsqueda del avión que escucharon caer en la isla, Chávez encuentra unos hongos y le pide a Evo Morales que los pruebe, ya que la cia los pudo plantar para atentar contra su vida.

— Hugo Chávez: ¡Evo! ¡Evo!, detente chico, estamos cansados y tenemos hambre. Mira Evo, tenemos comida.

— Evo Morales: no hay nada, señor. Eso solo son hongos.

- Hugo Chávez: Evo, pruébalos tú primero, no vaya ser que la cia los haya colocado allí y me envenene como un bobo. Un intento de magnicidio.

— Evo Morales: sí señor. No pasa nada.

- [Evo Morales prueba los hongos y alucina] (Min 2:28, Capítulo 5, Temporada 1).

Figura 4. La disputa (Isla Presidencial, Capítulo 5, Temporada 1)

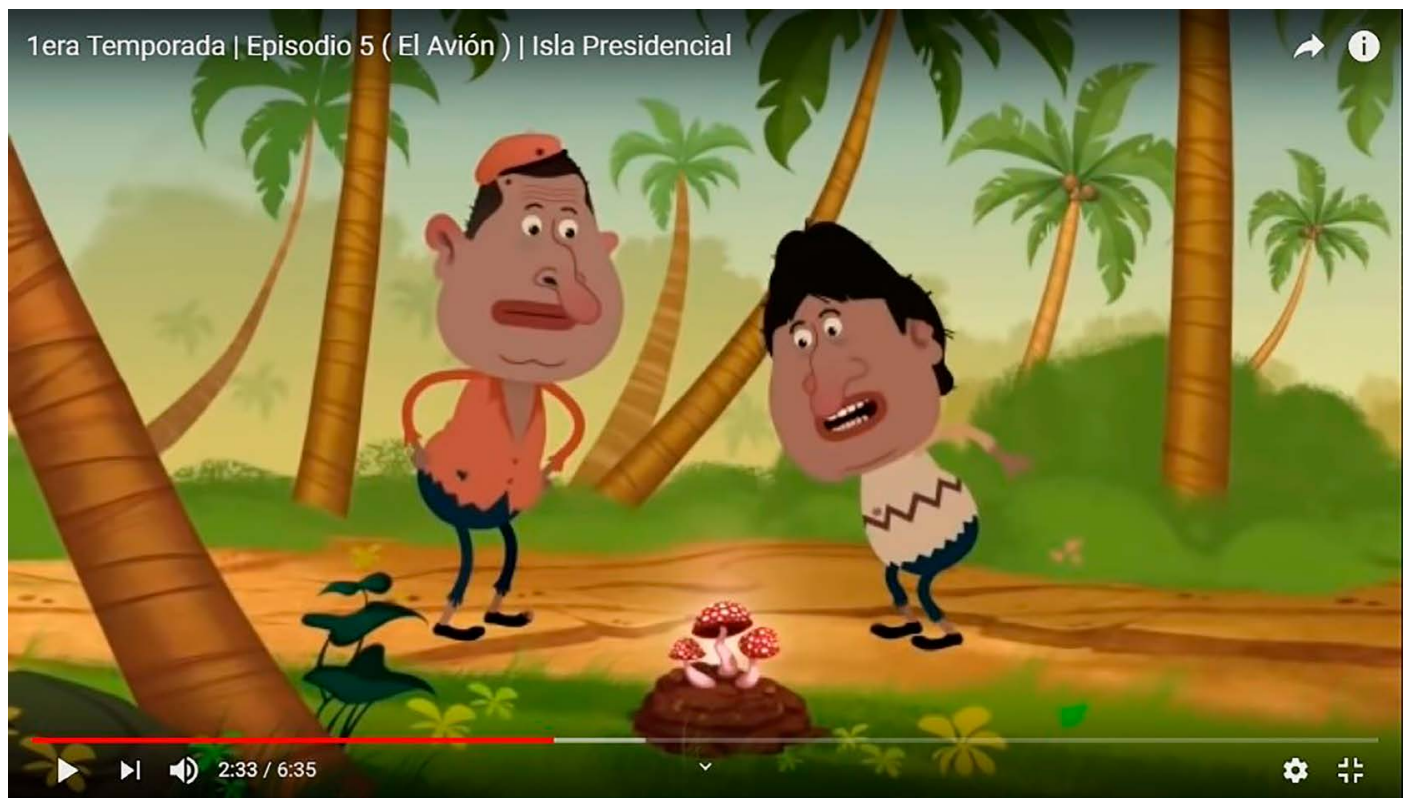

A la izquierda, Hugo Chávez y a la derecha, Evo Morales

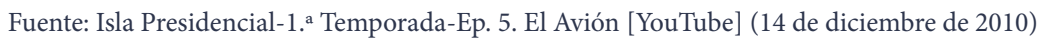

El personaje de Hugo Chávez en Isla Presidencial.

Un análisis de la sátira política a través de la teoríade las representaciones sociales de Serge Moscovici 
Dentro de la Isla Presidencial y durante su gobierno, se resaltan las conspiraciones creadas por Hugo Chávez, en las que involucra a personajes e instituciones para llevar a cabo sus acciones. Como ejemplo de lo anterior, aparece el personaje de Evo Morales como su principal soporte para la ejecución de estas, así la Organización de Estados Americanos aparece como sujeto de burlas y sátira, al considerarse una institución que tiene poca relevancia e incidencia dentro de los países que la conforman.

Las conspiraciones hacen parte del espectáculo político, porque se constituye de problemas sociales que surgen desde el discurso político, como se pudo ver durante el gobierno de Hugo Chávez en Venezuela, quien estableció un régimen socialista denominado Revolución Bolivariana, en el que prima el interés general sobre el particular, al realizar expropiaciones de bienes privados y destinar recursos petroleros para satisfacer parte de las necesidades que tenían las personas de escasos recursos en los sistemas de salud, educación y vivienda (Mercado, 2019). Esos problemas y personalidades proveen el contenido del periodismo político, desempeñan un papel central en la obtención de apoyo y oposición a las diversas causas políticas.

\section{5. ¡Chávez ya no soy yo! ¡Chávez es un pueblo!}

El expresidente Hugo Chávez se caracterizó por ser un líder carismático, por lograr el reconocimiento de los dominados, que lo involucra en un proceso emotivo. Según Molero (1995) es importante destacar lo puntualizado por Weber sobre el líder carismático:

El portador del carisma exige obediencia y adhesión en virtud de su misión, pero el carismático y su misión han de acreditarse por medio de éxitos que proporcionen beneficios a los seguidores. En caso contrario, el carisma podría llegar a perderse. La creación de un tipo de dominación carismática es siempre fruto de una situación extrema en el terreno político, económico o personal y origina una gran excitación que, siendo compartida por cierto número de personas, tiende a la consagración del heroísmo de cualquier clase que sea. Por tanto, la dominación carismática tiene un carácter profundamente emocional. (Molero, 1995, p. 45).

Además, Hugo Chávez en la mayor parte de los discursos que usó para su campaña política y durante su gobierno, utilizaba la frase: ¡Chávez ya no soy yo!, ¡Chávez es un pueblo! (Vicepresidencia de la República Bolivariana de Venezuela, 2019), en razón a esto, por medio de la serie audiovisual se hace burla a esta frase que usaba como una estrategia de poder y manipulación frente a la población venezolana.

La Constitución Republicana Bolivariana de Venezuela consagra la propiedad privada como principio y derecho en sus artículos 112 y 115; sin embargo, aclara que prima la función social de esta cuando se dedica al aprovechamiento de los recursos, es decir, a explotar el potencial de un bien. En ocasiones, el exmandatario venezolano en vida se refirió a que él nunca ha sido socialista sino comunista, teniendo en cuenta que al ser un gobierno comunista el derecho a la propiedad privada queda anulado, al igual que las clases sociales, prevaleciendo un gobierno social, en el que el sistema económico beneficia a todos por igual, debido a que se basa en la

El personaje de Hugo Chávez en Isla Presidencial.

Un análisis de la sátira política a través de la teoríade las representaciones sociales de Serge Moscovici 
productividad. No obstante, las decisiones de expropiar, las ejecutaba "a dedo" y solo bastaba con decir ¡Exprópiese!, se trataba de bienes que no estaban bajo el dominio y en poder del Gobierno.

En la primera temporada, episodio 4, llamado La balsa, los presidentes juegan un partido de fútbol para definir quiénes son los ganadores que podrán irse de la isla en la balsa, dividiéndose en dos grupos: entre los alineados y los no alineados. Durante el juego, el marcador iba a favor del grupo de los alineados, encabezado por el expresidente colombiano, lo cual desata una discusión entre Hugo Chávez y Álvaro Uribe Vélez, en el que el primero maneja una postura de forma dominante diciéndole al segundo:

— Hugo Chávez: exprópiese

— Álvaro Uribe Vélez: ¿Qué dijo?

- Hugo Chávez: ordeno que inmediatamente se expropie de esta cancha.

— Juan Carlos I: al parecer los presidentes discuten si el gol es o no válido, según el presidente Chávez, si la cancha es del pueblo, el gol técnicamente sería de él, porque bueno, Chávez es el pueblo.

— Álvaro Uribe Vélez: Chávez, usted es un bobo.

— Hugo Chávez: doble bobo.

- Álvaro Uribe Vélez: triple bobo.

— Hugo Chávez: vigésimo triple, doble bobo, doble bobo. (Min 1:48, Capítulo 4, Temporada 1).

Figura 5. Discusión (Isla Presidencial, Capítulo 4, Temporada)

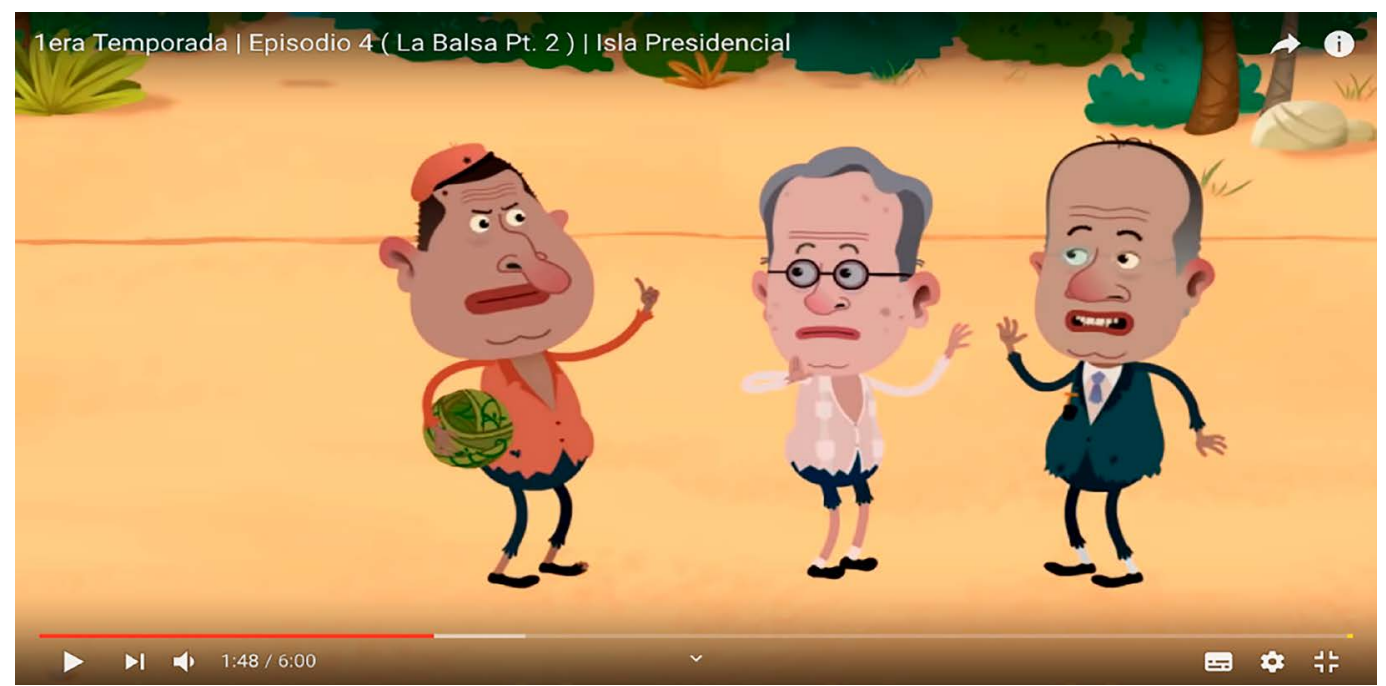

A la izquierda Hugo Chávez, en el centro Álvaro Uribe y a la derecha Felipe Calderón. Fuente: Isla Presidencial-1. Temporada-Ep. 4 La Balsa Pt. 2 [YouTube] (11 de julio de 2010) 


\section{El mesías}

Barack Obama y Hugo Chávez durante sus periodos de gobierno siempre manejaron buenas relaciones internacionales, acabando con la política "antiestadounidense", propia del gobierno chavista. Muchas veces se pudo ver el respeto que se tenía el uno al otro, a pesar de las inconformidades que tenía Obama frente a lo que estaba sucediendo en Venezuela para esa época en la que Hugo Chávez era presidente. Los dos exmandatarios siempre manifestaron y demostraron ser personas dispuestas al diálogo. Para Barack Obama, Hugo Chávez nunca fue una amenaza para su gobierno, ni para su país, mucho menos como un enemigo o rival, sino que siempre sostuvo que esas actitudes frente a esta persona eran con fines estratégicos para su gobierno y así dejar de dimensionar las problemáticas que existían antes, entre estos gobiernos (Infobae, 2016).

En este acápite queremos mostrar cómo el personaje de Hugo Chávez es representado como robot para ser manipulado por Barack Obama, de modo que este pueda realizar y controlar todo lo que sucede en la Isla Presidencial a través de él. Así mismo, observar que Hugo Chávez siempre tuvo la razón sobre sus teorías de la conspiración. Es por esto que Barack Obama aprovecha esta situación y lo convierte en el mesías, como el dador de todo lo que necesiten los demás presidentes para la supervivencia en la isla y lograr obtener el apoyo de todos, favoreciéndose con la situación en la que se encontraban. Para Juan Carlos I, Mariano Rajoy y Sebastián Piñera, las acciones que realizó el exmandatario siempre fueron cuestionadas. Por el contrario, Cristina Fernández, Rafael Correa y Evo Morales, tomaron estas acciones como milagros, pero sin duda para los demás son artimañas y formas de tener el control para lograr el poder sobre todos.

En la segunda temporada, episodio 6, llamado El Mesías, parte II, muestran a Hugo Chávez como el mesías, por dotarlos de agua potable tras días de sequía:

— Rafael Correa: Evo, ¿qué otra cosa puede hacer Chávez?

- Daniel Ortega: me parecería oportuno que solucionara ipso facto la problemática que vivimos con el vital líquido.

— José Mujica: no sé si estoy drogado, pero sigo sin entender ni una palabra de lo que dice.

- Evo Morales: creo que se refiere al agua, veré qué puedo hacer. Señor no hemos bebido agua en días, sus hijos de la izquierda imploramos que nos quite la sed.

- Hugo Chávez: negra Hipólita representa el amor y la generosidad desprendida de las madres, proceso de desalinización iniciando.

- (Como se ve en la imagen, el robot Chávez da de beber a los suyos).

— Evo Morales: ¡Es agua dulce!

- Cristina Fernández: ¿No hay otra manera de beber agua?

El personaje de Hugo Chávez en Isla Presidencial.

Un análisis de la sátira política a través de la teoríade las representaciones sociales de Serge Moscovici 
— Evo Morales: no cuestione los métodos del gran líder, jummm.

— Juan Carlos I: ¡gilipollas! No podéis idolatrar a alguien como si fuera el elegido de Dios, ni que fuera un rey.

- Juan Manuel Santos: ¿seguros de que no podemos tomar un sorbito? (Min 1:21, Capítulo 6, Temporada 2. El Mesías parte 2).

Figura 6. El Mesías Robot. Isla Presidencial, Capítulo 5, Temporada 2.

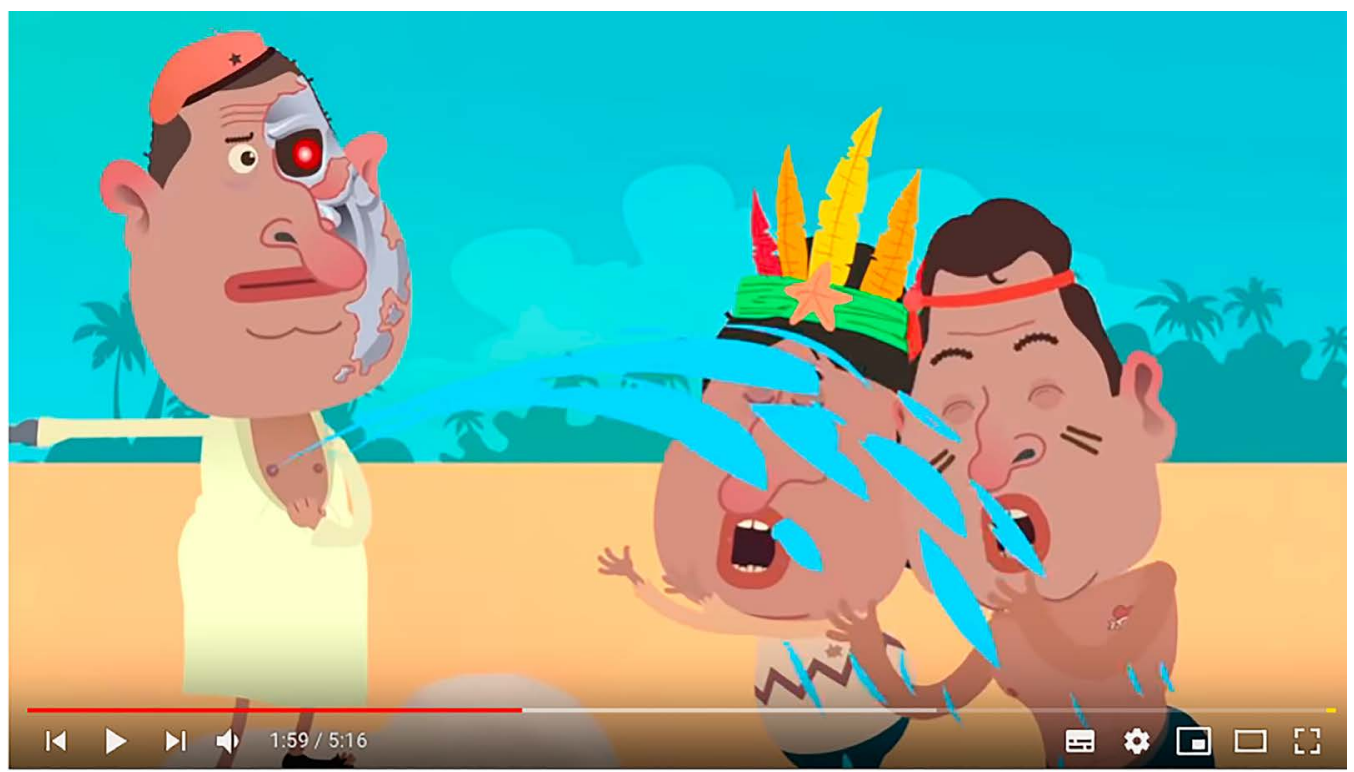

De izquierda a derecha: Hugo Chávez, Evo Morales, Rafael Correa

Fuente: Isla Presidencial- 2.a Temporada - Ep. 2. El Mesías Pt. 2 (15 de octubre de 2018)

Varios presidentes aceptan las acciones que realiza Chávez. Queda claro el alcance del poder que tiene Hugo Chávez como el dador de todo lo que necesiten para sobrevivir, al dotarlos de alimento y agua. A pesar de que todos los personajes que hacen parte de la serie ostentan poder, el cual se resalta bajo circunstancias de necesidad, en la que los demás deben ceder parte de este para sobrevivir. En este escenario siempre hay quienes aprovechan y utilizan su poder como capacidad de hacer real lo posible (Marina, 2008).

\section{Resultados y discusiones}

En esta investigación se identificaron las representaciones sociales del poder que se ven presentes a través del personaje del exmandatario venezolano Hugo Chávez. Este se destacó en su gobierno y en la serie audiovisual, por tener una ideología socialista y una postura antimperialista. Las representaciones sociales del poder se ven inmersas a través de su discurso 
dominante, el lenguaje que empleaba durante los episodios relacionados, las características de un líder carismático y las relaciones de poder que utilizaba con los otros mandatarios de la serie Isla Presidencial.

La Teoría de las representaciones sociales de Serge Moscovici (2000) se utilizó para contrastar las representaciones sociales de poder dentro de los subcapítulos desarrollados en el presente escrito. Hugo Chávez, el Mesías, es el análisis del primer campo de representación: la información, en la que se derivan los estereotipos que se construyen desde el personaje de Hugo Chávez en Isla Presidencial y a su vez, desde la comunidad que lo eligió como presidente de la República de Venezuela.

Por otra parte, la segunda dimensión es el campo de representación, en el cual se organiza el contenido de la representación en forma jerarquizada. Martín Mora (2002), en su trabajo La teoría de las representaciones sociales de Serge Moscovici alude que esta dimensión "permite visualizar el carácter del contenido, las propiedades cualitativas o imaginativas, en un campo que integra informaciones en un nuevo nivel de organización en relación a sus fuentes inmediatas" (p. 10). Cabe resaltar, que se debe estudiar el objeto de análisis en su totalidad, de acuerdo a que: "Debe analizarse en función de la totalidad del discurso sobre un objeto y no solo en un párrafo o en una frase" (p. 10).

Asimismo, Denise Jodelet establece que se debe realizar un estudio a partir del contenido representativo; es decir, en primer lugar, todo lo que constituye las representaciones y como último, las estructuras a través de las cuales se reflejan los sistemas de representación; en razón a esto el presente estudio será la base para determinar el sentido, lo que significa, los procesos y las formas de organización del contenido. (Jodelet, 1989). El Mesías proyecta la segunda dimensión, porque no solo surge como sátira dentro de la serie audiovisual, sino que es la adjudicación que le otorga la comunidad a Chávez como un personaje de poder que introduce el cambio positivo en su sociedad.

El primer y segundo subcapítulo: Érase una vez volcanes, cocos y monos imperialistas y Chávez vs. Imperialismo, constituyen el tercer campo de representación, la actitud, allí el personaje de Hugo Chávez toma una posición crítica frente al sistema imperialista/capitalista, al igual que una fuerte posición de rechazo hacia los presidentes que lideran países con el mismo sistema, especialmente al exmandatario colombiano Álvaro Uribe Vélez.

En Conspiraciones se pudo ver que el papel fundamental que empleó Hugo Chávez para crear imaginaciones sobre un mundo imperialista, la ausencia de vida en Marte por causa del capitalismo y lo ocurrido el 9/11, aludiendo que todo fue planeado por el expresidente Bush. Esto como resultado de la dimensión del campo de representación, en la que se debe tener en cuenta el discurso empleado, el estudio a partir de las premisas y los sistemas que lo representan. Por el contrario, en ¡Chávez ya no soy yo! ¡Chávez es un pueblo!, se encuentra la dimensión de la información, en su gobierno hizo alusión constante a esta frase durante sus discursos y quiso demostrar con su imagen que su gobierno siempre estaría al servicio del pueblo, en busca de

El personaje de Hugo Chávez en Isla Presidencial.

Un análisis de la sátira política a través de la teoríade las representaciones sociales de Serge Moscovici 
beneficios para las personas con bajos recursos económicos, conforme a su ideología. De igual forma, durante los episodios de la serie audiovisual al expropiar el balón de fútbol demuestra los fines de dicha ideología política.

El texto abarcó diferentes estrategias de búsqueda del poder, presentes en el personaje $\mathrm{Hu}$ go Chávez de la serie Isla Presidencial. De esta manera, en primera medida se analizó cómo las ideologías políticas dividen a los personajes en dos grupos que suelen estar en constante conflicto. Por medio de las conspiraciones, entablan relaciones de poder y emergen situaciones para alcanzar la autoridad que cada personaje anhela tener. La conspiración dentro de la serie audiovisual fue utilizada como herramienta para alcanzar el poder; un ejemplo de ello, cuando logró ganar las elecciones presidenciales de la isla. No obstante, las conspiraciones fueron un instrumento clave para la obtención y preservación del poder en su carrera política; hay quienes afirman que el "Caracazo" fue una conspiración de Hugo Chávez.

La imagen de Hugo Chávez representó un líder carismático, un presidente que pretende ser del pueblo y considerado como el mesías por varios personajes de la isla, especialmente con quienes compartió su ideología. En vida, fue el salvador o mesías del pueblo venezolano; de acuerdo al reportaje de la ввс (Olmo, 2018), un testimonio de un seguidor afirma: "La situación en 1998 era de auténtico desastre y él pudo presentarse como un salvador en medio de ese desastre porque los venezolanos ya no creían en nadie de los partidos políticos tradicionales" (s. p.).

Considerado como mesías y caracterizado por ser un líder dominante, autoritario y del pueblo, se le adjudica su lema: ¡Chávez no soy yo! ¡Chávez es el pueblo! Su mandato fue representado por su facultad de expropiar los bienes de propiedad privada para ser utilizados en beneficio de la comunidad; así como lo intentó hacer durante la serie audiovisual, al querer expropiar la balsa que no le pertenecía. A su vez, este lema hace parte de las estrategias que usa como discurso político para mantener el poder.

En Isla Presidencial, logra convencer a Evo Morales como un discípulo que profesa obediencia, lealtad y sumisión. Con el poder no solo se crea sumisión, sino que también hay resistencia y rebeldía. Sin embargo, Marina (2008) en su libro La pasión del poder. Teoría y práctica de la dominación, diferencia la rebeldía de la resistencia, porque la primera no solo se encarga de dificultar el ejercicio del poder, sino que se enfrenta a él. Es así que su poder se ve limitado ante las actuaciones de inconformidad de los demás personajes, especialmente aquellos que se caracterizaron por representar la corriente ideológica política de derecha.

A través del personaje de Hugo Chávez se evidencian las representaciones sociales del poder por medio de las estrategias y situaciones creadas por él, que permitieron determinar la fascinación por adquirir y saciar la sed de poder.

El personaje de Hugo Chávez en Isla Presidencial.

Un análisis de la sátira política a través de la teoríade las representaciones sociales de Serge Moscovici 


\section{Referencias}

Edelman, M. (1991). La construcción del espectáculo político. Buenos Aires, Argentina: Ediciones Manantial.

El Heraldo (15 de septiembre de 2016,). ¿Quién es Jaime Garzón? Recuperado de https://www.elheraldo. co/nacional/quien-es-jaime-garzon-285291

Expansión.mx (22 de marzo de 2011). Hugo Chávez: El capitalismo habría acabado con la vida en Marte. Recuperado de https://expansion.mx/mundo/2011/03/22/hugo-chavez-el-capitalismo-habria-acabado-con-la-vida-en-marte

Graziani, O., \& Ravell, J. (Productores). (11 de julio de 2010). Isla Presidencial-1a Temporada-Ep. 4. La Balsa Pt. 2 [Serie audiovisual YouTube]. Recuperado de https://www.youtube.com/watch?v=_ Hp5KKLAAOw\&t=144s

Graziani, O. \& Ravell, J. (Productores) (14 de diciembre de 2010). Isla Presidencial-1a Temporada-Ep. 5. El Avión [Serie audiovisual YouTube]. Recuperado de https://www.youtube.com/watch?v=AAjPDM7SNE\& $=238 \mathrm{~s}$

Graziani, O. \& Ravell,J.(Productores) (5 deoctubre de 2018). Isla Presidencial2a Temporada-Ep. 1.El Volcán [Serie audiovisual YouTube]. Recuperado de https://www.youtube.com/watch?v=fQOC3xZM9fI

Graziani, O. \& Ravell, J. (Productores) (15 de octubre de 2018). Isla Presidencial- 2a TemporadaEp. 5. El Mesías Pt. 1. [Serie audiovisual YouTube]. Recuperado de https://www.youtube.com/ watch?v=V3s2lxwjywk

Gómez, S. (2012). Chávez se mueve hacia el comunismo con disfraz de demócrata. El Tiempo. Recuperado de: https://www.eltiempo.com/archivo/documento/CMS-12297716

Hinkelammert, F. J. (2003). Pensar en alternativas: Capitalismo, socialismo y la posibilidad de otro mundo. Digitalizado por Biblioteca 'P. Florentino Idoate, S.J.' Universidad Centroamericana José Simeón Cañas. Recuperado de http://hdl.handle.net/11674/3044

Infobae (10 de marzo de 2016). Barack Obama dice que no trató a Hugo Chávez como enemigo y eso "ayudó a EEUu en la región". Recuperado de https://www.infobae.com/2016/03/11/1796298-barackobama-dice-que-no-trato-hugo-chavez-como-enemigo-y-eso-ayudo-eeuu-la-region/

Jodelet, D. (1989). Représentations sociales: Un domaine en expansion. En D. Jodelet (coord.), Les représentations sociales (pp. 47-78). París, Francia: Presses Universitaires de France.

La Vanguardia. (23 de marzo de 2011,). Hugo Chávez sospecha que el capitalismo acabó con la vida en Marte. [Redacción]. Recuperado de https://www.lavanguardia.com/internacional/20110323/54131430897/ hugo-chavez-sospecha-que-el-capitalismo-acabo-con-la-vida-en-marte.html

Lenin, V. I. (2003). El imperialismo fase superior del capitalismo. Recuperado de https://www.fundacionfedericoengels.net/images/PDF/lenin_imperialismo.pdf

Marina, J. A. (2008). La pasión del poder. Teoría y práctica de la dominación. Barcelona, España: Editorial Anagrama.

Mercado, L. (23 de enero de 2019). Cinco posibles causas que llevaron a Venezuela a la crisis. El Tiempo. Recuperado de https://www.eltiempo.com/mundo/venezuela/causas-que-llevaron-a-venezuela-ala-crisis- 84652

El personaje de Hugo Chávez en Isla Presidencial.

Un análisis de la sátira política a través de la teoríade las representaciones sociales de Serge Moscovici 
Molero, F. (2016). El estudio del carisma y del liderazgo carismático en las ciencias sociales: Una aproximación desde la psicología social. Revista de Psicología Social, 10 (1), 43-60. https://doi. org/10.1174/021347495763835256

Mora, M. (2002). La teoría de las representaciones sociales de Serge Moscovici. Athenea Digital, 2, 1-25. https://doi.org/10.5565/rev/athenead/v1n2.55

Moscovici, S. (1961). El psicoanálisis, su imagen y su público. Paris, Francia: Presses Universitaires de France. Recuperado de: https://www.researchgate.net/publication/266257708_El_psicoanalisis_su_ imagen_y_su_publico

Moscovici, S. (2000). The History and Actuality of Social Representations. En Duveen, G. (org). Social Representations. Explorations in Social Psychology (pp. 120-155). Cambridge: Polity Press.

Olmo, G. (6 de diciembre de 2018). Triunfo de Hugo Chávez en 1998: Cómo era la Venezuela en la que triunfó Chávez hace 20 años (y en qué se parece a la actual). BBC. Recuperado de https://www.bbc. com/mundo/noticias-america-latina-46463299

Real Academia Española (s. f.). Definición de Conspirar. Recuperado de https://dle.rae.es/conspirar

Ulloa, C. (2008). Arma del contrapoder: Humor político y medios. Chasqui, Revista Latinoamericana de Comunicación, (104), 72-77. Recuperado de https://dialnet.unirioja.es/descarga/articulo/5791261. pdf

Valero Heredia, A. (2014). Libertad de expresión y sátira política: Un estudio jurisprudencial. Revista Internacional de Historia de la Comunicación, 2(1), 86-96. https://doi.org/10.12795/RiHC.2014.i02.05

Vicepresidencia de la República Bolivariana de Venezuela (2019). Chávez no soy yo, Chávez es el pueblo. [Especial]. Recuperado de http://www.vicepresidencia.gob.ve/especial-chavez-no-soy-yo-chavezes-el-pueblo/ 